This is the post peer-review accepted manuscript of:

A. Al-Rimawi and D. Dardari, "Characterization of link lifetime in the presence of random blocking objects," 2017 IEEE International Conference on Communications (ICC), Paris, 2017, pp. 1-6.

doi: https://doi.org/10.1109/ICC.2017.7997186

The published version is available online at:

http://ieeexplore.ieee.org/stamp/stamp.jsp?tp=\&arnumber=7997186\&isnumber=7996317

(C) 2017 IEEE. Personal use of this material is permitted. Permission from IEEE must be obtained for all other uses, in any current or future media, including reprinting/republishing this material for advertising or promotional purposes, creating new collective works, for resale or redistribution to servers or lists, or reuse of any copyrighted component of this work in other works 


\title{
Characterization of Link Lifetime in the Presence of Random Blocking Objects
}

\author{
Ashraf Al-Rimawi ${ }^{\dagger}$ Member, IEEE and Davide Dardari ${ }^{\ddagger}$ Senior, IEEE \\ Department of Electrical and Computer Engineering ${ }^{\dagger}$, Birzeit University, Palestine \\ DEI ${ }^{\ddagger}$, University of Bologna, via Venezia 52, 47023 Cesena (FC), Italy \\ Email: aalrimawi@birzeit.edu ${ }^{\dagger}$,davide.dardari@unibo.it ${ }^{\dagger}$
}

\begin{abstract}
The presence of obstacles between a mobile user and the serving access point might determine frequent link blockages that could be expensive in terms of signaling caused by handovers and antenna beamforming, especially in the perspective of millimeter wave communications. In this paper, an analytical framework to characterize the statistics of the link lifetime of a moving user in the presence of random obstacles with different sizes is derived for two different mobility models. Using statistical geometry arguments, closed-forms of the cumulative distribution function and the average link lifetime are obtained as a function of the distance, user's speed and direction, obstacles' density and size. The analytical framework is validated through simulations and allows to get insights on the impact of system parameters on the link lifetime.
\end{abstract}

\section{INTRODUCTION}

Link blockage can arise due to the presence of big obstacles, such as walls or cars in vehicular ad hoc networks, but also small obstacles like furniture and people in a crowded scenario might determine serious link blockages especially at millimeter waves $(\mathrm{mmW})$. Together with user's mobility, blockages due to obstacles may force frequent handovers between access points (APs) and consequent signaling overhead or connection drops, especially if narrow beamforming is performed as expected in future $\mathrm{mmW}$ wireless systems. This could have a strong impact especially on device-to-device (D2D) or vehicular communications. As a consequence, such a phenomenon has received particular attention in the scientific community. Specifically, in addition to experimental campaigns [1], many progresses have been made in the analytical characterization of the coverage probability in the presence of obstacles to get insights on the key parameters affecting the performance [2][3].

In prior work, most of papers related to blockage models focus on characterizing the coverage probability for static wireless networks [4]-[6]. In [4] the authors proposed a stochastic framework to model random blockages in urban cellular networks and analyze the performance of cellular networks in urban areas. In [5] the coverage and capacity of $\mathrm{mmW}$ cellular systems are studied with focus on special factors such as the limited scattering nature of $\mathrm{mmW}$ channels and beam steering to provide highly directional transmission with limited hardware complexity. In [6] the authors proposed framework model based on stochastic geometry to characterize

This research was supported by Birzeit Universityt (Grant 604050), Palestine and the European H2020 project XCycle (Grant 635975).

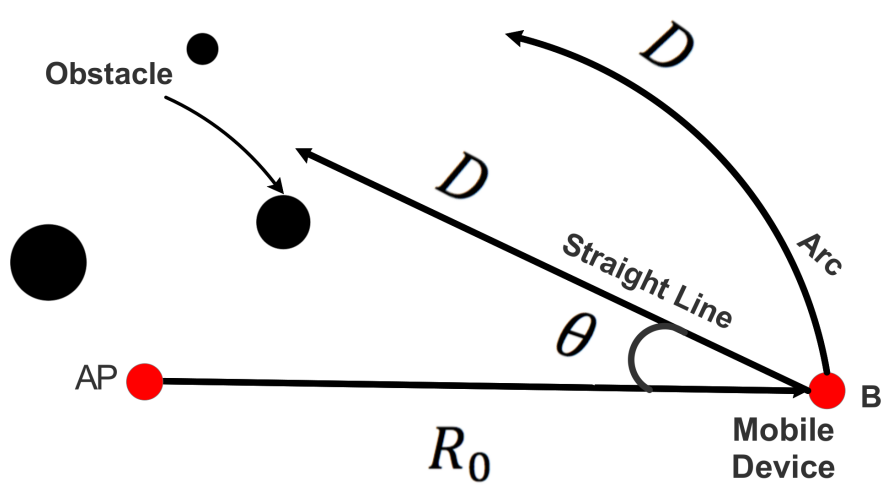

Fig. 1: Mobility Model

one and two-way signal-to-interference plus noise ratio (SINR) of $\mathrm{mmW}$ ad hoc networks with directional antennas, random blockages, and ALOHA channel access. The results show that $\mathrm{mmW}$ networks in the presence of blockage can support much higher densities and larger spectral densities compared with lower frequency communication. Moreover, the results indicate that the rate coverage of $\mathrm{mmW}$ can be much greater than lower frequency due to the increased bandwidth.

Unfortunately, for dynamic mobility models, coverage probability does not provide information about link blockages duration/rate as its evaluation usually does not include spatial/time correlation of the link state. Link lifetime is the proper figure of merit that should be introduced and characterized in order to account for user's mobility. In particular, link lifetime is defined as the time elapsed until a blockage or an out-ofrange (i.e., distance larger than the transmission range) are encountered when the mobile user is traveling along a certain path.

While many progresses have been made in the analytical characterization of coverage probability, less results considering the link lifetime are available so far. In [7] the authors provided a set of requirements necessary to define a mobility metric and investigated the effect of link lifetime on the performance of mobile ad hoc networks. In [8] the probability distribution for the link distance between two randomly mobile radios is presented by considering two different deployment scenarios for the mobile locations, whereas in [9] the impact of radio channels and node mobility on link dynamics in wireless networks is studied. In all cases no obstacles are considered.

In this context the aim of this paper is to fill the gap in 


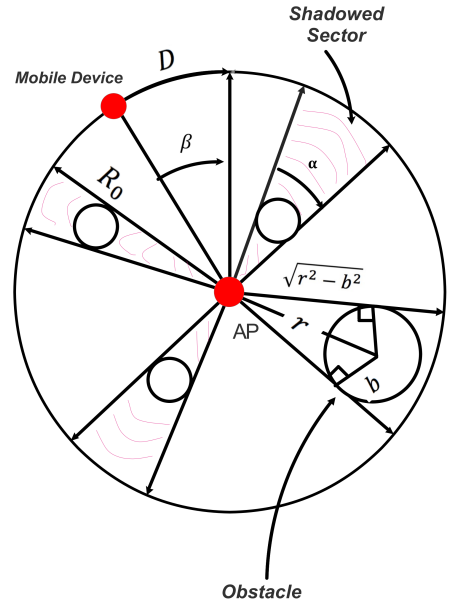

(a) Circle Scenario

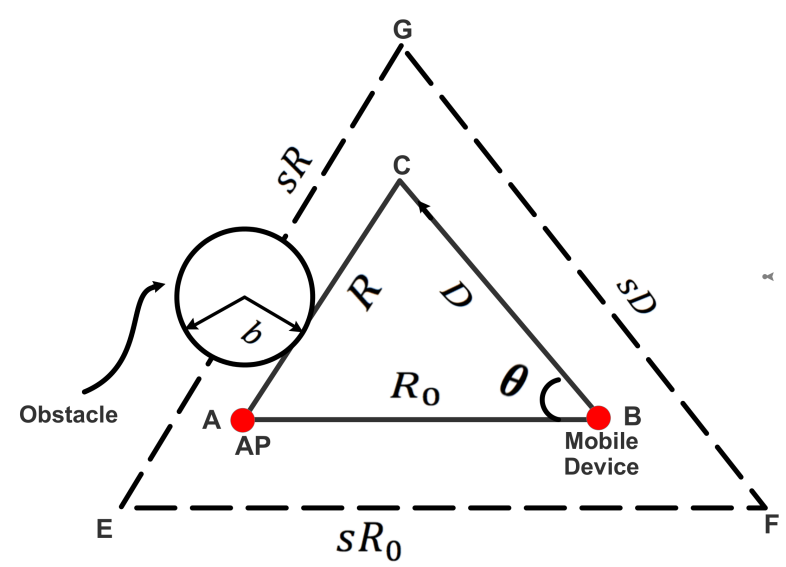

(b) Straight Line Scenario; where $s$ is the scale factor

Fig. 2: Scenarios of a wireless link between a mobile device and AP

characterizing the link lifetime by proposing a new analytical framework (for dynamic mobility model) able to account for both users mobility and effects of blockages. In other words, we address for the first time, based on our best knowledge, the problem of characterizing the statistics of the link lifetime in the presence of random blocking objects. Specifically, we consider one of the most realistic scenario where we assume a mobile device moving along the straight line with constant speed. Further, a random number of obstacles located in random positions with different sizes is present. We derive closed-form expressions for the statistical distribution of link lifetime accounting for the shadowing effect caused by obstacles depending on their dimension and distance from the AP. In the numerical results we investigate the joint effect of the main system parameters such as the density and size of obstacles and the communication distance, from which network design guidelines can be easily obtained. Analytical results are compared with simulations with the purpose to assess their validity.

\section{SySTEM MOdel}

As shown in Fig. 1 we consider a random number of objects (obstacles) distributed according to a Poisson point process (PPP) in a plane. Objects are completely blocking as usually happens when working at $\mathrm{mmW}$ using beamforming techniques. Since we are only interested in the shadowing effect of obstacles when located in between the mobile device and the AP, we approximate them by circles. The assumption might be a good approximation in the $\mathrm{mmW}$ wireless networks in which the reflections are weak due to the higher carrier frequency, and signals are highly directive with the deployment of adaptive antenna arrays at both transmitters and receivers [4]. To account for obstacles with different sizes, a number $n$ of classes of objects with radius $b_{i}, i=1,2, \ldots, n$ distributed according to PPP and density $\lambda_{i}$ are considered. The following scenarios for the wireless link between the mobile device and AP are of interest:
- Circle Mobility Model: As shown in Fig. 1 we consider a wireless link between a mobile device moving for $D$ meters at constant speed along a circle of radius $R_{0}$ from the serving AP located in the center. This model approximates situations where the radial movement is small compared to $R_{0}$ and/or $D$. Note that only objects inside the circle of radius $R_{0}$ might affect the link between AP and mobile device.

- Straight Line Mobility Model: As shown in Fig. 1 a more realistic scenario considers the mobile device moving for $D$ meters along a straight line with constant speed starting from point $B$ (Mobile Device) with angle $\theta$.

The link between the mobile device and the AP is established if the mobile device is located in visible region, that is, it is outside all shadow sections determined by the objects. Now define the link drop condition as the event in which the mobile device when moving falls inside at least one shadow region. The link lifetime $T_{L}$ is the time elapsed from the initial position until the first link drop condition occurs. The purpose is to characterize the statistics of the link lifetime as a function of $\lambda_{i}, b_{i}, D, \theta$ and the initial distance $R_{0}$ for both mobility models.

\section{Characterization of the Link Lifetime}

\section{A. Circle Model Scenario}

As shown in Fig. 2(a) we consider the mobile device moving within a sector of angle $\beta=\frac{D}{R_{0}} \leq \pi$ at distance $R_{0}$ from the AP with linear speed $v$. In the presence of one object located in a random position within the circle $R_{0}$, the probability that the shadow sector of angle $\alpha$, created by the object, intersects the sector $\beta$ defined by the mobile device is

$$
\mathbb{P}(\text { shadow } \mid \alpha)=\frac{\beta+\alpha}{2 \pi} .
$$

We start the analysis by considering a single object of radius $b$ located at distance $r$ from the AP. As shown in Fig. 2(a), 
the angle $\alpha$ of the shadow sector can be calculated by using simple trigonometric rules, where

$$
\tan \left(\frac{\alpha}{2}\right)=\frac{b}{\sqrt{\left(r^{2}-b^{2}\right)}} \quad r>b .
$$

When $r \gg b$ (small obstacles or large distances), (2) can be approximated as $\tan \left(\frac{\alpha}{2}\right) \approx \frac{b}{r}$. The accuracy of this approximation will be assessed by simulation in the numerical results. According to the PPP distribution of objects, the number of objects belonging to the $i$ th class that fall inside the circle of radius $R_{0}$ is Poisson distributed with mean $\lambda_{i} \pi R_{0}^{2}$ [10]. Their positions are independent, identically distributed (i.i.d.) with uniform distribution so that the probability density function (p.d.f.) of their distance $r$ from the origin is given by

$$
f_{r}(r)=\frac{2 r}{R_{0}^{2}}
$$

where $r \in\left[0, R_{0}\right]$, zero otherwise. Their angle distribution is uniform in $[0,2 \pi)$.

Under the approximation of (2) and given (3), the cumulative distribution function $(\mathrm{CDF})$ of random variable (RV) $\alpha$ results

$$
\begin{aligned}
F_{\alpha}(x)=\mathbb{P}(\alpha \leq x) & =1-\mathbb{P}\left(r \leq \frac{b}{\tan \left(\frac{x}{2}\right)}\right) \\
& =1-\frac{b^{2}}{R_{0}^{2} \tan ^{2}\left(\frac{x}{2}\right)}
\end{aligned}
$$

for $\alpha \in\left[2 \tan ^{-1}\left(\frac{b}{R_{0}}\right), \pi\right]$, zeros otherwise where $\mathbb{P}(E)$ denotes the probability of event $E$. The corresponding p.d.f. is

$$
f_{\alpha}(\alpha)=\frac{b^{2} \sin (\alpha)}{2 R_{0}^{2} \sin ^{2}\left(\frac{\alpha}{2}\right)}
$$

defined in the same interval. From (5) the expected value of $\alpha$ results

$$
\varepsilon(\eta)=\mathbb{E}[\alpha]=\eta(-\eta \pi+2)+2\left(\eta^{2}+1\right) \tan ^{-1}(\eta) .
$$

Note that $\varepsilon(\eta)$ depends only on the ratio $\eta=b / R_{0}$. The unconditional probability is

$$
\begin{aligned}
P_{1}(\beta, \eta)=\mathbb{P}(\text { shadow }) & =\mathbb{E}_{\alpha}[\mathbb{P}(\text { shadow } \mid \alpha)] \\
& =\frac{\beta}{2 \pi}+\frac{\mathbb{E}[\alpha]}{2 \pi}=\frac{\beta}{2 \pi}+\frac{\varepsilon(\eta)}{2 \pi}
\end{aligned}
$$

which is valid for $0 \leq \beta \leq \pi$. Since there are $n$ classes of objects, each with a random number of objects according to the Poisson distribution, the probability that a mobile device moving in a sector of angle $\beta$ at distance $R_{0}$ is not shadowed by any object is

$$
\begin{aligned}
P_{0}^{(\operatorname{arc})}(\beta) & =\prod_{i=1}^{n} \sum_{k=0}^{\infty}\left[1-P_{1}\left(\beta ; \eta_{i}\right)\right]^{k} \frac{\left(\pi \lambda_{i} R_{0}^{2}\right)^{k}}{k !} e^{-\pi \lambda_{i} R_{0}^{2}} \\
& =\exp \left(-\sum_{i=1}^{n} \frac{\lambda_{i} R_{0}^{2} \beta}{2}\right) \exp \left(-\sum_{i=1}^{n} \frac{\lambda_{i} R_{0}^{2} \varepsilon\left(\eta_{i}\right)}{2}\right) .
\end{aligned}
$$

Supposing the mobile device moves along the circle at constant linear speed $\nu$, it turns out that the CDF of the link lifetime $T_{\mathrm{L}}$ is

$$
P_{L}^{(a r c)}\left(T_{L}\right)=\mathbb{P}\left(T \leq T_{L}\right)=1-P_{0}^{(a r c)}\left(\frac{\nu T_{L}}{R_{0}}\right)
$$

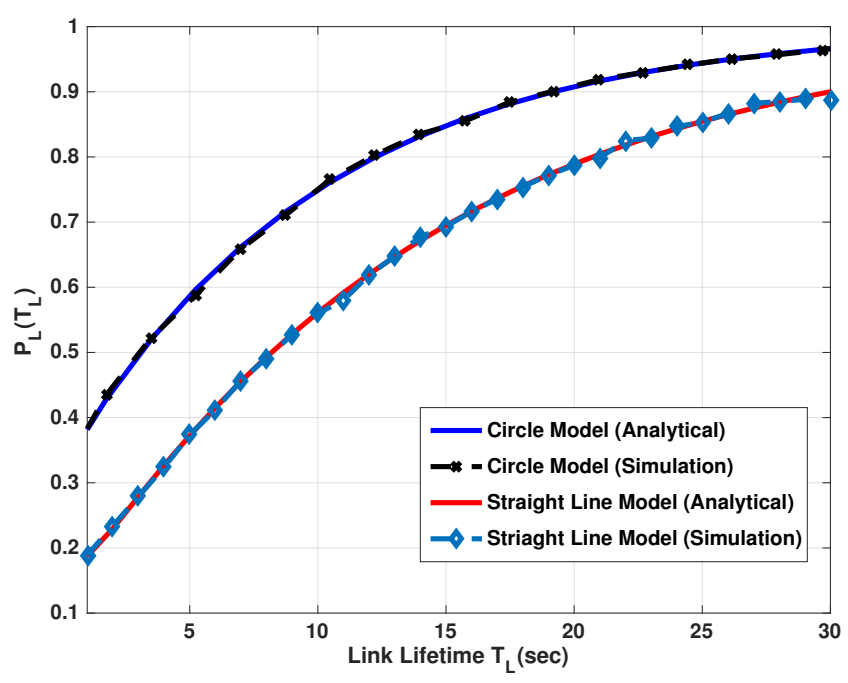

Fig. 3: Comparison between a user moving on a circle and on the corresponding straight line. One class of obstacles: $b_{1}=$ $1 \mathrm{~m}, \lambda_{1}=0.01$ Blocks $/ \mathrm{m}^{2}, R_{0}=20 \mathrm{~m}$ and $\theta=\pi / 4$.

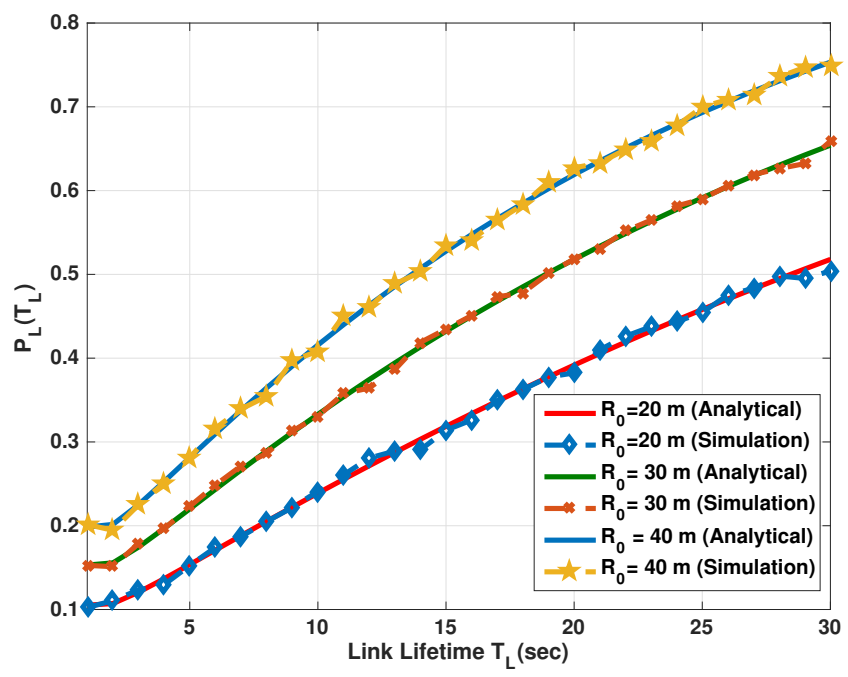

Fig. 4: Impact of link initial distance $R_{0}$ on the link lifetime CDF (Straight Line Model). Two classes of obstacles: $b_{1}=$ $3 \mathrm{~m}, b_{2}=1 \mathrm{~m}, \lambda_{1}=0.001$ Blocks $/ \mathrm{m}^{2}, \lambda_{2}=0.002$ Blocks $/ \mathrm{m}^{2}$ and $\theta=\pi / 4$.

being $\nu T_{L}=D$.

From (8) we can note that the first term of $P_{0}^{(\operatorname{arc})}(\beta)$ gives the probability that no shadows are present in a sector of angle $\beta$ when considering punctiform objects. In fact $\exp \left(-\pi \lambda_{i} R_{0}^{2} \beta / 2\right)$ represents the probability that no obstacles of class $i$ have their center in the sector of angle $\beta$. The second term accounts for object's dimension and its distance distribution and depends on the ratio $\eta_{i}=b_{i} / R_{0}$ through (6) and not on $\beta$. This is an interesting result which tells that the effect of object's size is not a function of the distance traveled by the mobile device. From the previous analysis the average link lifetime $T_{A}$ can be derived by differentiating (9) 


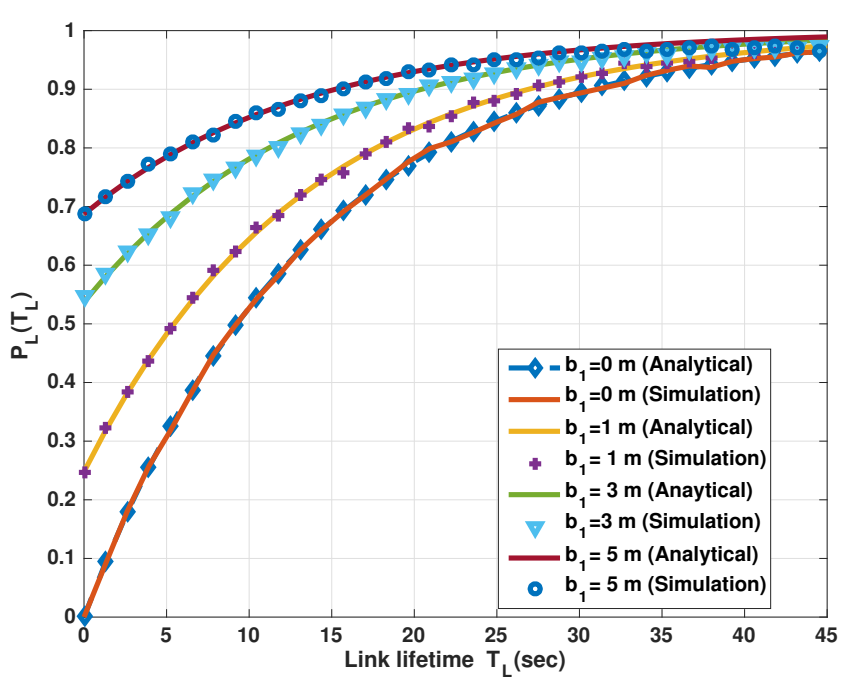

Fig. 5: Impact of obstacle's size on the link lifetime (Circle Model). One class of obstacles: $R_{0}=15 \mathrm{~m}$, and $\lambda_{1}=0.01$ Blocks $/ m^{2}$.

with respect to $T_{L}$ and taking the expectation resulting in

$$
T_{A}=\sum_{i=1}^{n} \frac{2}{\lambda_{i} R_{0} \nu} \exp \left(-\sum_{i=0}^{n} \frac{\lambda_{i} R_{0}^{2}}{2} \varepsilon\left(\eta_{i}\right)\right) .
$$

The derivation can be found in Appendix A.

\section{B. Straight Line Scenario}

As shown in Fig.2(b) we consider the mobile device moving along the straight line $\mathrm{BC}$ with constant speed $v$ and angle $\theta$. The mobile device will keep in contact with the AP for the entire trip from $\mathrm{B}$ to $\mathrm{C}$ if no object or part of it falls inside the communication region which is defined by the triangle $\mathrm{ABC}$. Given an obstacle of radius $b$, it falls inside the communication region if its center is located inside the augmented triangle EFG as shown in Fig.2(b) .

Since we consider there are $n$ classes of objects distributed according to PPP, then the probability that no object falls inside the communication region can be expressed as

$$
\begin{aligned}
P_{0}^{(l i n)}(D) & =\mathbb{P}(\text { no obstacles in } \triangle \mathrm{EFG}) \\
& \approx \exp \left(-\sum_{i=1}^{n} \lambda_{i} A\left(D ; s_{i} ; R_{0}, \theta\right)\right),
\end{aligned}
$$

where (11) is approximated since there could be some obstacles with centers located at the edges that do block the link. Fortunately the probability of this event is very low as confirmed by simulations., $A\left(D ; s_{i} ; R_{0}, \theta\right)$ is the area of triangle EFG whose derivation can be found in appendix B, and $s_{i}$ is a scale factor which is given by (18).

Supposing the mobile device moves at constant speed $v$, it turns out that the CDF of the link lifetime $T_{L}$ is

$$
P_{L}^{(\operatorname{lin})}\left(T_{L}\right)=\mathbb{P}\left(T \leq T_{L}\right)=1-P_{0}^{(\operatorname{lin})}\left(\nu T_{L}\right),
$$

being $\nu T_{L}=D$.

From the previous analysis the average link lifetime $T_{A}$ can be derived by differentiating (12) with respect to $T_{L}$ and taking the expectation resulting in

$$
\begin{aligned}
T_{A}= & \sum_{i=1}^{n} \frac{2 b_{i} R_{0}}{2 b_{i}+R_{0} \sin (\theta)} \\
& \times \exp \left(\sum_{i=1}^{n}-b_{i} \lambda_{i}\left(2 b_{i} \csc (\theta)+R_{0}\right)\right) \\
& K_{1}\left(\lambda_{i} b_{i} \csc (\theta)\left(2 b_{i}+R_{0} \sin (\theta)\right)\right)
\end{aligned}
$$

where $K_{1}($.$) is the Modified Bessel function of the Second$ kind.

\section{Numerical Results}

Using the proposed analytical framework, in this section we investigate how the link lifetime in a wireless network is affected by the presence of random blocking obstacles. Moreover, we present some Monte Carlo simulation results to validate our analytical framework. For the sake of illustration and without loss of generality we study the effects of two classes of blocking objects $(n=2)$ if not otherwise specified and we normalize the link lifetime in both proposed models with respect to $\nu$. i.e., $\nu=1$, so that numerically it corresponds to $D$.

In Fig. 3 the accuracy of our model when used to characterize a user moving along a straight line is presented and compared with the circle scenario in term of $P_{L}\left(T_{L}\right)$. From the results it can be seen that the circle scenario leads to a significant worse performance compared with a user moving along the straight line (more realistic scenario) and provides an upper bound of the link lifetime.

In Fig. 4 we study the effect of the initial distance $R_{0}$ on $P_{\mathrm{L}}\left(T_{\mathrm{L}}\right)$ for the straight line scenario. As expected, the initial distance has a significant impact on the link lifetime. In fact, given a fixed obstacles' density, the higher the distance the higher is the probability to encounter obstacles in between the mobile user and the AP. The effect of obstacle's size is shown in Fig. 5 in which it is evident that the error one could commit when approximating obstacles with points $\left(b_{1}=0\right)$ could be significant. The good matching with simulation results validates the accuracy of the analytical framework and the approximation of (2) even when $b$ is not much smaller than $r$. The behavior of the average link lifetime for the circle model for one class of obstacles as a function of obstacle's size $b_{1}$ by varying $\lambda_{1}$ is shown in Fig. 6. Results indicate that the impact of nodes' density is higher with small objects. In fact the average link lifetime decreases exponentially with respect to the density of obstacles and object's size according to different relationship as evident in (6) and (10).

\section{Conclusions}

In this paper we have presented a stochastic analysis of the communication link lifetime between a mobile device and its serving AP in the presence of $n$ classes of randomly located objects characterized by different densities and sizes. We have derived closed-form expressions of the link lifetime CDF and corresponding average, which indicate the exponential behavior of the link lifetime as a function of obstacle's size 


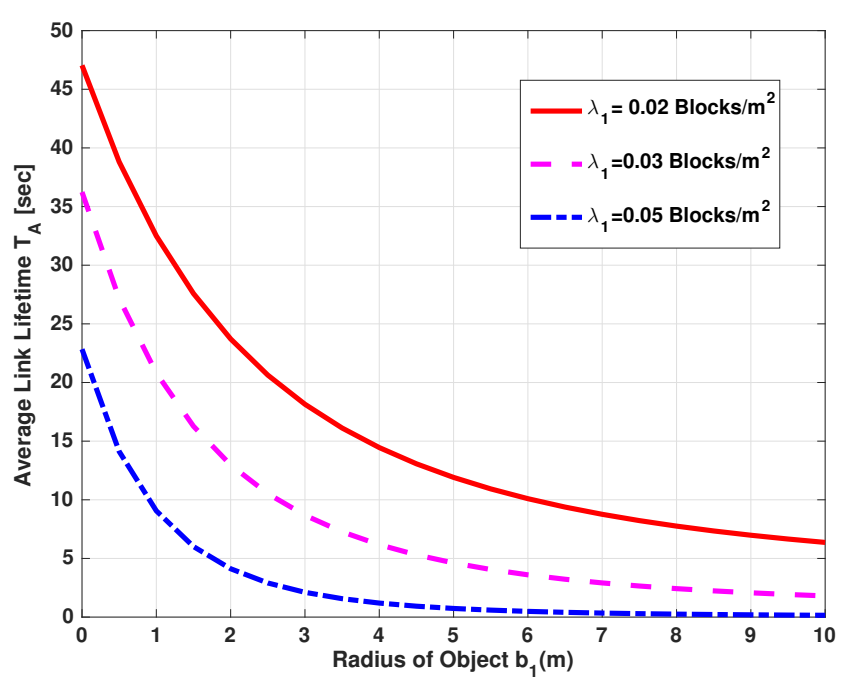

Fig. 6: Impact of density of obstacles on the average link lifetime (Circle Model). One class of obstacles: $R_{0}=10 \mathrm{~m}$.

and allowed to get insights on the interplay between obstacles' density, dimension, and user's distance. Comparisons with simulation results have confirmed the validity of the analytical framework.

\section{APPENDIX A}

\section{DERIVATION OF THE AVERAGE LINK LIFE TIME $T_{A}$ FOR THE CiRCle Mobility MOdel}

By differentiating (9) with respect to $T_{L}$ then the p.d.f. of link lifetime $p_{L}^{(a r c)}\left(T_{L}\right)$ can be expressed as

$$
\begin{aligned}
p_{L}^{(\text {arc })}\left(T_{L}\right) & =\sum_{i=1}^{n} \frac{\lambda_{i} R_{0} \nu}{2} \exp \left(-\sum_{i=1}^{n} \frac{\lambda_{i} R_{0}^{2} \beta}{2}\right) \\
& \times \exp \left(-\sum_{i=1}^{n} \frac{\lambda_{i} R_{0}^{2} \varepsilon\left(\eta_{i}\right)}{2}\right) .
\end{aligned}
$$

From (14), the average link lifetime $T_{A}$ can be derived

$$
T_{A}=\mathbb{E}\left[T_{L}\right]=\int_{0}^{\infty} T_{L} p_{L}^{(a r c)}\left(T_{L}\right) d T_{L}
$$

where the final result can be found in (10) .

\section{APPENDIX B \\ DERIVATION OF THE AREA OF TRIANGLE EFG \\ SHOWN IN FIG. 2}

As shown in Fig.7 we are interested in scaling the inner triangle $\mathrm{ABC}$ with the scale factor $s$ in order to obtain the area of outer triangle EFG. In fact, based on geometric consideration, the area of the triangle EFG is given by

$$
A\left(s, R_{0}, D, \theta\right)=s^{2} A_{i n}\left(R_{0}, D, \theta\right),
$$

where $A_{i n}\left(R_{0}, D, \theta\right)=\frac{1}{2} R_{0} D \sin (\theta)$ is the area of triangle $\mathrm{ABC}$.

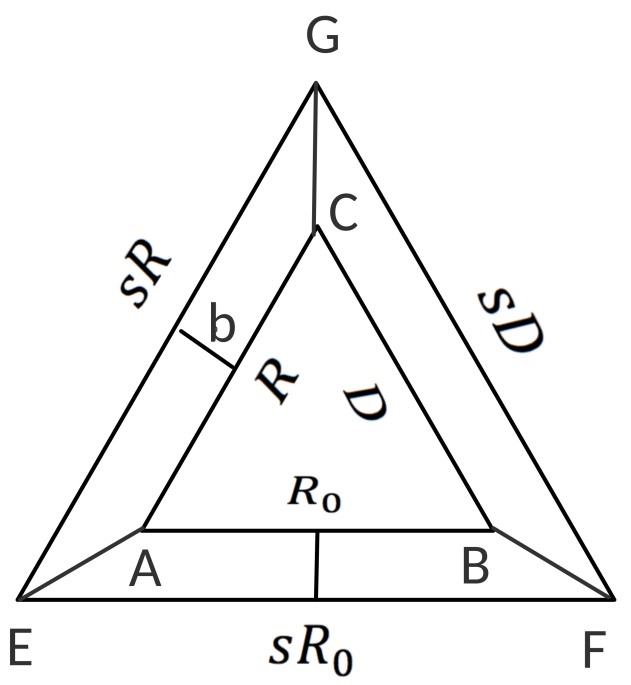

Fig. 7: The geometric analysis of area of triangle EFG

On the other hand, the left side of (16) can also be expressed as

$$
\begin{aligned}
A\left(s, R_{0}, D, \theta\right) & =\frac{1}{2} R_{0} D \sin (\theta)+\frac{1}{2} b\left(R_{0}+s R_{0}\right) \\
& +\frac{1}{2} b(D+s D)+\frac{1}{2} b\left(R_{1}+s R_{1}\right) \\
& =A_{i n}\left(R_{0}, D, \theta\right)+\frac{1}{2} b P_{i n}\left(R_{0}, D, \theta\right) \\
& +\frac{1}{2} s b P_{i n}\left(R_{0}, D, \theta\right),
\end{aligned}
$$

where $P_{i n}\left(R_{0}, D, \theta\right)$ is the perimeter of triangle ABC.

By substituting (17) in (16) the scale factor $s$ results

$$
s=\frac{b \csc (\theta)}{D}+\frac{2 b \csc (\theta)}{R_{0}}+1 .
$$

Finally, by substituting (18) in (16) the area of triangle EFG can be obtained.

\section{REFERENCES}

[1] M. R. Akdeniz, Y. Liu, M. K. Samimi, S. Sun, S. Rangan, T. S. Rappaport, and E. Erkip, "Millimeter wave channel modeling and cellular capacity evaluation," IEEE Journal on Selected Areas in Communications, vol. 32, no. 6, pp. 1164-1179, June 2014.

[2] T. Bai and R. W. Heath, "Coverage analysis for millimeter wave cellular networks with blockage effects," in 2013 IEEE Global Conference on Signal and Information Processing (GlobalSIP), Dec 2013, pp. 727-730.

[3] H. Shokri-Ghadikolaei and C. Fischione, "The transitional behavior of interference in millimeter wave networks and its impact on medium access control," IEEE Transactions on Communications, vol. 64, no. 2 , pp. 723-740, Feb 2016.

[4] T. Bai, R. Vaze, and R. W. Heath, "Analysis of blockage effects on urban cellular networks," IEEE Transactions on Wireless Communications, vol. 13, no. 9, pp. 5070-5083, Sept 2014.

[5] S. Akoum, O. El Ayach, and R. Heath, "Coverage and capacity in mmwave cellular systems," in 2012 Conference Record of the Forty Sixth Asilomar Conference on Signals, Systems and Computers (ASILOMAR), Nov 2012, pp. 688-692.

[6] A. Thornburg, T. Bai, and R. W. H. Jr., "Performance analysis of mmwave ad hoc networks," CoRR, vol. abs/1412.0765, 2014. [Online]. Available: http://arxiv.org/abs/1412.0765

[7] S. Shelly and A. Babu, "Probability distribution of link life time in vehicular ad hoc networks," in 2013 International Conference on Advances in Computing, Communications and Informatics (ICACCI), Aug 2013, pp. 1418-1423. 
[8] J. Boleng, W. Navidi, and TracyCamp, "Metrics to enable adaptive protocols for mobile ad hoc networks," in Int.Conf. on Wireless Networks (ICWN2002), Aug 2002, pp. 293-298.

[9] L. E. Miller, "Distribution of link distance in a wireless network," Journal of Research of National Institute of Standards and Technology, vol. 106, April 2001.

[10] A. Zanella, M. Stramazzotti, F. Fabbri, E. Salbaroli, D. Dardari, and R. Verdone, "Comments on "probability distributions for the number of radio transceivers which can communicate with one another"," IEEE Transactions on Communications, vol. 46, no. 5, pp. 1287-1289, May 2009. 